\title{
Evaluación de ELISA F29 como marcador de eficacia del tratamiento etiológico en la enfermedad de Chagas
}

\author{
DIANA FABBRO*, ELSA VELÁZQUEZ**, NORBERTO MENDOZA*, MIRTHA STREIGER*, ENRIQUE \\ ARIAS*, SUSANA DENNER*, MONICA DEL BARCO*, NORBERTO AMICONE*, CARLOS PRAVIA** \\ NORA MALAGRINO**, y ANDRES MARIANO RUIZ**
}

\section{ASSESSMENT OF ELISA F29 AS A MARKER OF ETIOLOGICAL TREATMENT EFFICACY FOR CHAGAS DISEASE}

A transversal study was performed on sera from chronic chagasic patients treated with nifurtimox $(\mathrm{Nx})$ or benznidazole $(\mathrm{Bz})$, in order to evaluate the Trypanosoma cruzi flagellar calcium-binding protein (F29) as a marker for therapeutic effectiveness. An ELISA was used with these F29 recombinant antigen, and its relation to conventional serology (CS) and parasitological and clinical evolution was analysed. Sera from 118 patients with retrospective, serological, parasitological and clinical information was available, were analyzed. Patients were grouped into: A) 30 treated patients whose CS became negative after treatment; B) 34 treated patients whose CS remained positive; C) 54 untreated patients. A double-blind trial was conducted simultaneously in all serum samples, by means of CS (indirect hemagglutination, direct agglutination and indirect immunofluorescence) and ELISA F29. The ELISA F29 test was non reactive in: $100 \%$ of group A, $82.4 \%$ of group B and $13 \%$ of group C. The infected patients who presented electrocardiographic alterations compatible with chronic chagasic myocardiopathy $(n=11)$ were reactive for ELISA F29. All patients whose parasitological studies (xenodiagnosis and/or strout method) were positive presented a high reactivity to the ELISA F29 test. The correlation between ELISA F29 and CS was statistically significant $(p<005)$ in the treated group whose CS was non reactive (group A) and the untreated group (group C). As opposed to this, in the group of treated patients whose CS remained positive (group B), the ELISA F29 test was reactive only in a 17.6\%.These results suggest that the fast and user-friendly ELISA F29 test could be useful to monitor changes after trypanocidal treatment.

Key words: Chagas disease, serological marker of therapeutic efficacy.

\section{INTRODUCCIÓN}

La infección por Trypanosoma cruzi, agente causal de la enfermedad de Chagas, fue descubierta hace más de 90 años. El tratamiento tripanocida es eficaz en etapas tempranas de la infección. Para la etapa crónica la eficacia del mismo sigue en discusión.

El nifurtimox $(\mathrm{Nx})$ y el benznidazol $(\mathrm{Bz})$ demostraron ser útiles en su acción tripanocida en pacientes infectados en la fase aguda y en niños con enfermedad de Chagas por transmisión connatal tratados tardíamente (antes de los 2 años de edad $)^{1,2}$, si bien se ha comprobado la

\footnotetext{
* Centro de Investigaciones Endemias Nacionales (CIEN), Facultad de Bioquímica y Ciencias Biológicas, Universidad Nacional del Litoral, Santa Fe.

** Instituto Nacional de Parasitología Dr. Mario Fatala Chaben, Buenos Aires.
} 
existencia de cepas de T. cruzi resistentes a alguna de las drogas ${ }^{3-5}$. Estudios más recientes realizados en niños menores de 13 años en período indeterminado de la enfermedad de Chagas, mostraron buena tolerancia y alto porcentaje de eficacia del $\mathrm{Bz}$, utilizando en la evaluación métodos serológicos no convencionales ${ }^{6-8}$.

La caracterización de la eficacia del tratamiento antiparasitario específico en pacientes adultos con infección crónica por T. cruzi no está definido actualmente, debido a que la serología convencional (SC) permanece reactiva en la gran mayoría de los pacientes, aún muchos años después de finalizado el tratamiento tripanocida $\mathrm{y}$, los métodos parasitológicos tradicionales (xenodiagnóstico, hemocultivo) tienen escasa sensibilidad en esta etapa de la infección. Además la evolución clínica de la enfermedad de Chagas es de lenta progresión y un elevado porcentaje de pacientes infectados permanece, aún sin tratamiento, en el período indeterminado, sin manifestaciones electrocardiográficas y radiológicas.

Por otra parte, los mecanismos por los cuales los pacientes con infección crónica desarrollan enfermedad no son completamente conocidos. En el período agudo de la infección el rol del parásito en el proceso inflamatorio está bien definido. Para la fase crónica de la enfermedad se ha planteado la posibilidad de una patogenia autoinmune, aunque existen dudas acerca de que el mecanismo autorreactivo sea el único responsable de las lesiones cardíacas. La persistencia del parásito generaría una reactividad inflamatoria crónica, y ésta induciría una respuesta inmune cuyo blanco sería el propio tejido del huésped ${ }^{9-11}$.

Estos antecedentes demuestran la necesidad de contar con herramientas sensibles y específicas que permitan monitorear la eficacia del tratamiento de manera segura y eficiente.

La proteína flagelar ligadora de calcio ha sido utilizada como marcador temprano de cura de la enfermedad de Chagas ${ }^{12,13}$. Sosa Estani y col $^{6}$, evaluaron la eficacia del tratamiento antiparasitario específico en niños entre 6 y 12 años, con infección por T. cruzi tratados con benznidazol. Para ello implementaron una técnica de ELISA usando como antígeno una molécula recombinante obtenida de una proteína flagelar del parásito (conocida como F29) ${ }^{14}$.

El objetivo de este trabajo fue evaluar un ensayo inmunoenzimático con la proteína recombinante F29 (ELISA F29), como posible marcador serológico de eficacia terapéutica, en sueros de pacientes con infección crónica por $T$. cruzi tratados y no tratados, analizando su relación con la SC, la parasitemia y los aspectos clínicos.

\section{MATERIAL Y MÉTODOS}

Diseño: estudio transversal, con información retrospectiva de una cohorte.

Se estudiaron 118 sueros de pacientes infectados con T. cruzi provenientes del C.I.E.N (Centro de Investigaciones sobre Endemias Nacionales). De todos ellos se disponía de información clínica, serológica y parasitológica durante un seguimiento de hasta 23 años, residiendo siempre en la ciudad de Santa Fe (zona de baja endemicidad). Los pacientes fueron controlados anual o bianualmente, mediante exámenes clínicos complementados con electrocardiograma y radiografía de tórax. Conjuntamente se realizaba toma de muestra de sangre para realizar SC para Chagas. Los controles parasitológicos, mediante xenodiagnóstico ${ }^{15}(\mathrm{Xd})$, se realizaron en períodos de tiempo mayores. Las muestras de sueros analizadas en el presente trabajo son las correspondientes al último control de los pacientes.

Sesenta y cuatro sueros correspondían a pacientes que habían recibido tratamiento tripanocida (37 niños y 24 adultos con infección crónica y 3 niños menores de un año con infección congénita) y 54 sueros de infectados crónicos que permanecían sin tratar (17 niños y 37 adultos) (Tabla 1 ).

El tratamiento antiparasitario específico en los pacientes crónicos adultos se administró con anterioridad al año $1983^{16}$, ya que recién a partir de esa fecha las Normas Nacionales indicaron que no se debía tratar a los infectados crónicos ${ }^{17}$. La dosis y tiempo de tratamiento fueron: nifurtimox $(\mathrm{Nx}) 12-15 \mathrm{mg} / \mathrm{kg} /$ día para niños y $8-10 \mathrm{mg} / \mathrm{kg} /$ día para adultos, durante 45-60 días; benznidazol $(\mathrm{Bz}): 5 \mathrm{mg} / \mathrm{kg} /$ día por 30 días para niños y adultos. Diecinueve pacientes recibieron tratamiento con $\mathrm{Nx}$ y 45 con $\mathrm{Bz}$.

Tenían Xd previo 47 pacientes: 30 tratados con controles parasitológicos pre y posttratamiento y 17 no tratados. En 5 niños menores de 4 años, en los que se sospechó infección aguda, se utilizó el método de Strout. 
La Tabla 1 muestra los diferentes grupos estudiados según edad, tiempo de seguimiento, droga utilizada en los tratados y resultados de la $\mathrm{SC}$.

Los pacientes se clasificaron en 3 grupos, según hubieran recibido o no tratamiento y según el resultado de la SC:

Grupo A. 30 pacientes tratados con negativización de la SC post-tratamiento. Todos eran asintomáticos. Este grupo estuvo compuesto por: 23 niños infectados crónicos con un seguimiento post-tratamiento de 14,6 \pm 4,8 años; 2 niñas con infección aguda transplacentaria y 5 adultos chagásicos crónicos con $19 \pm 2$ años de seguimiento post-tratamiento.

Grupo B. 34 pacientes tratados que no negativizaron la SC post-tratamiento. Este grupo estuvo compuesto por: 14 niños infectados crónicos asintomáticos con seguimiento de 2,5 $\pm 1,8$ años post-tratamiento; 1 niña con infección congénita tratada con nifurtimox a los 2 meses de vida y, 19 adultos infectados crónicos con seguimiento post-tratamiento de $19,5 \pm 2,8$ años. En este grupo, excepto un paciente, todos permanecieron asintomáticos.

Grupo C. 54 infectados chagásicos crónicos no tratados compuesto por: 17 niños asintomáticos sin seguimiento y 37 adultos (6 con miocardiopatía chagásica crónica -MChCr-al inicio del seguimiento y 4 enfermaron durante el mismo) seguidos durante $17 \pm 3,9$ años. La SC fue persistentemente positiva en 30 pacientes adultos mientras en 7 de ellos presentó fluctuaciones en los diferentes controles serológicos.

Grupo Control: Se utilizaron 35 muestras de sueros sin anticuerpos anti-T. cruzi como controles no reactivos (NoR) para todas las pruebas serológicas.

\section{Exámenes de laboratorio}

Se utilizaron reacciones de SC como hemaglutinación indirecta (HAI), aglutinación directa (AD) con y sin 2 mercaptoetanol ante sospecha de infección chagásica aguda, e inmunofluorescencia indirecta (IFI) con antiinmunoglobulina humana total. Se consideró reactivo cuando por lo menos 2 de 3 reacciones serológicas presentaron títulos mayores o iguales a $1 / 32$.

Se procesaron simultáneamente a doble ciego, los 118 sueros correspondientes al último control de la población descripta conjuntamente con las 35 muestras de suero de no infectados, nuevamente mediante las reacciones serológicas cuantitativas mencionadas anteriormente $\mathrm{y}$, por primera vez, con el test de ELISA utilizando como antígeno la proteína recombinante F29 (ELISA F29).

Tabla 1. Clasificación de infectados chagásicos según edad, tratamiento, serología convencional y tiempo de seguimiento

\begin{tabular}{|c|c|c|c|c|c|c|c|c|c|c|c|c|}
\hline \multirow{3}{*}{\multicolumn{2}{|c|}{ Edaden años }} & \multirow{2}{*}{\multicolumn{5}{|c|}{$\begin{array}{l}\text { Tratados }(n=64) \\
\text { Grupo A }(n=30) \\
\text { SC Final NoR }\end{array}$}} & \multicolumn{6}{|c|}{ No tratados $(n=54)$} \\
\hline & & & & & & & \multicolumn{3}{|c|}{$\begin{array}{c}\text { Grupo B }(n=34) \\
\text { SC Final R }\end{array}$} & \multirow[t]{2}{*}{$\begin{array}{c}\text { Grupo C } \\
\text { SC R }\end{array}$} & \multirow[t]{2}{*}{$\mathbf{t} \pm \mathbf{d s}$} & \multirow[t]{2}{*}{ Total } \\
\hline & & $\mathbf{N x}$ & $\mathbf{B z}$ & Total & $\mathbf{t} \pm \mathbf{d s}$ & $\mathbf{N x}$ & $\mathbf{B z}$ & Total & $\mathbf{t} \pm \mathbf{d s}$ & & & \\
\hline \multirow[t]{2}{*}{$\leq 14$} & $\leq 7$ & 4 & $15^{*}$ & 19 & $14,6 \pm 4,8$ & $1 * *$ & 4 & 5 & $2,5 \pm 1,8$ & 3 & 0 & 27 \\
\hline & $7-14$ & 0 & 6 & 6 & & 0 & 10 & 10 & & 14 & & 30 \\
\hline \multirow[t]{3}{*}{$>14$} & $15-29$ & 0 & 1 & 1 & $19 \pm 2$ & 6 & 2 & 81 & $19,5 \pm 2,8$ & 16 & $17,1 \pm 3,9$ & 25 \\
\hline & $30-44$ & 2 & 0 & 2 & & 4 & 6 & 10 & & 17 & & 29 \\
\hline & $\geq 45$ & 1 & 1 & 2 & & 1 & 0 & 1 & & 4 & & 7 \\
\hline \multicolumn{2}{|c|}{ Total } & 7 & 23 & 30 & & 12 & 22 & 34 & & 54 & & 118 \\
\hline
\end{tabular}

La edad de los pacientes corresponde al momento que recibieron tratamiento y en los no tratados al comienzo del estudio de seguimiento; $\mathrm{t} \pm \mathrm{ds}$ : tiempo promedio de seguimiento (en años) \pm desvío standard; SC: serología convencional; R: reactiva; NoR: no reactiva; Bz: benznidazol; Nx: nifurtimox.

* En este grupo de pacientes 2 niñas con infección connatal fueron tratadas antes del año de vida.

** Niña con infección congénita tratada a los 2 meses de vida, la muestra del suero aquí analizada corresponde a los 10 meses de edad. 
Antigeno F29: La secuencia de la proteína recombinante F29 fue obtenida a partir del clon pMalp2X F29 ${ }^{14}$ mediante PCR utilizando la polimerasa $P f u$ y los primers específicos:

5'-ATGGGTGCTTGTGGGTCGAA-3' y 5'TCAAGCCTTCTCCGGCACGT-3. El fragmento amplificado fue purificando con el kit Wizard SV Gel and PCR Clean-Up System (Promega) y posteriormente subclonado en el sitio Sma I del vector $\mathrm{pQE} 32$. La proteína $6 \mathrm{His}-\mathrm{F} 29$ fue expresada y purificada utilizando una columna de ácido nitrilotriacético- níquel (Ni-NTA, Qiagen) siguiendo el protocolo del fabricante.

ELISA F29: El ensayo se realizó como ya se describió anteriormente ${ }^{6}$, con la diferencia que se utilizó la proteína 6His-F29 en lugar de la MBP-F29. La proteína purificada se resuspendió en buffer carbonato $\mathrm{pH}$ 9,6 con una concentración $5 \mu \mathrm{g} / \mathrm{ml}$ y en un volumen final de $50 \mu \mathrm{l}$ por pocillo. Se acopló a placas de poliestireno de 96 pocillos Immuno Plate Maxisorp Surface (Nunc) durante toda la noche a $4^{\circ} \mathrm{C}$. Las placas se bloquearon con PBS-5\% leche durante 1 hora a temperatura ambiente.

La dilución de los sueros se realizó en PBS$1 \%$ leche en una concentración 1/200. La incubación de los sueros se realizó durante una hora a $37^{\circ} \mathrm{C}$.

Luego de tres lavados con PBS-Tween 0.05\% se incubó con el segundo anticuerpo: anti-IgG humano acoplada a la enzima peroxidasa (DAKO, diluidos 1/7.500 en PBS-leche al 1\% p/v).

Luego de lavar, el revelado se realizó con ofenilenediamina dihidrocloruro (OPD) $0,4 \mathrm{mg} /$ $\mathrm{ml}$ en una solución de buffer citrato $\mathrm{pH}$ : 5 con $0,024 \%$ de $\mathrm{H}_{2} \mathrm{O}_{2}$. La DO a $490 \mathrm{~nm}$ fue medida en un lector de microplacas.

El título de corte fue de 0.200 DO.

\section{Análisis estadístico}

Prueba de Shapiro-Wilk. Coeficiente de correlación por rangos de Spearman. Prueba de $\chi^{2}$ de Pearson. Para todas las pruebas se fijó un nivel de significación $\mathrm{p}=0,05$.

\section{RESULTADOS}

En la Figura 1 se observan los resultados de ELISA F29 en los diferentes grupos estudiados.

Grupo A: el 100\% (30/30) fue NoR para ELISA F29. Pre-tratamiento se demostró la presencia del parásito en 18 pacientes: una niña con infección congénita de 4 meses de vida presentó Strout positivo y 17 infectados crónicos tuvieron $\mathrm{Xd}(+)$. Los controles parasitológicos realizados post-tratamiento en estos 17 pacientes fueron persistentemente negativos, con un promedio de $5 \mathrm{Xd}$ /paciente durante 10 años

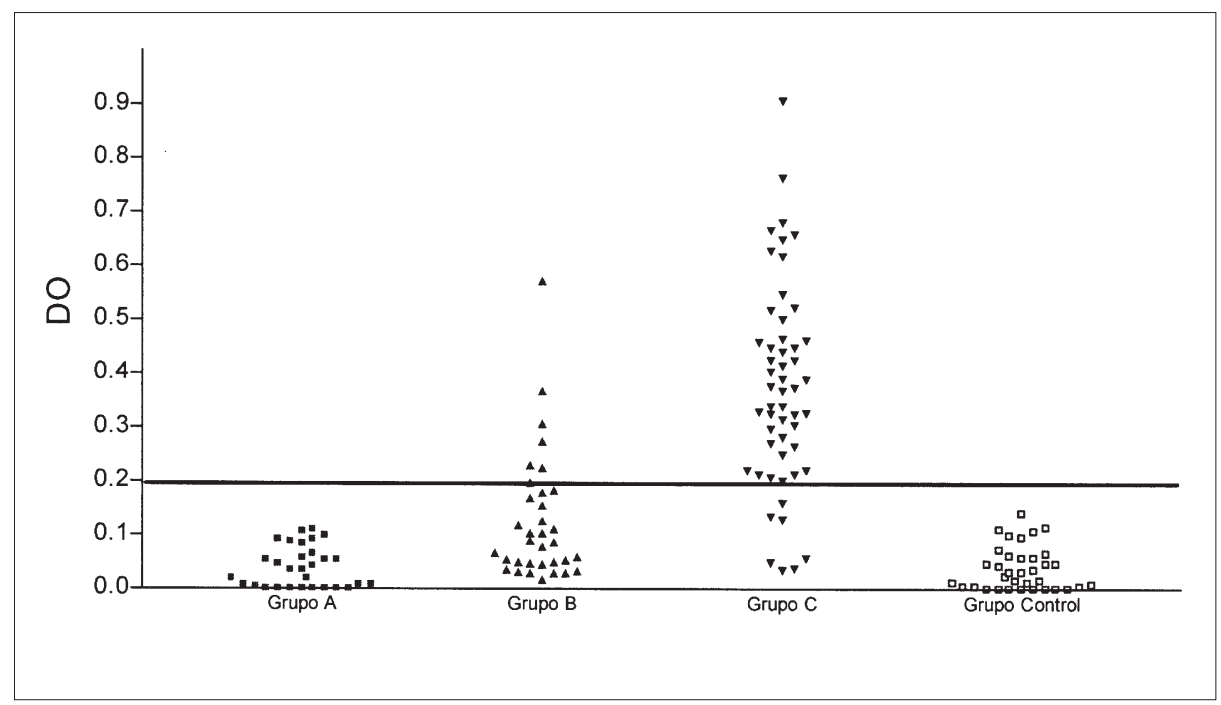

Figura 1. Resultados de las lecturas (DO) para la reacción de ELISA F29 en infectados chagásicos con y sin tratamiento antiparasitario específico. Grupo A: 30 pacientes tratados con serología convencional final no reactiva; Grupo B: 34 pacientes tratados con serología convencional final reactiva; Grupo C: 54 pacientes que no recibieron tratamiento; Crupo Control: 35 personas no infectados con T. cruzi; DO: lectura en densidades ópticas (valor de corte 0,200). 
posteriores a la finalización de la quimioterapia específica. En este grupo todos los pacientes $(100 \%)$ permanecieron sin manifestaciones clínicas ni electrocardiográficas compatibles con miocardiopatía chagásica durante $19 \pm 2$ años de seguimiento en los adultos y $14,6 \pm 4,8$ años en los niños con infección crónica.

Grupo B: el 82,4\% (28/34) fue NoR para ELISA F29. Durante el seguimiento, un solo paciente presentó alteraciones electrocardiográficas compatibles con miocardiopatía chagásica crónica (MChCr).

En este grupo, se observa un punto superior alejado del conjunto $(\mathrm{DO}=0,571)$ correspondiente a una muestra de suero analizada a los 10 meses post-tratamiento, de una niña con infección congénita tratada con $\mathrm{Nx}$ a los 2 meses de vida, que presentó falla terapéutica (Strout positivo y serología con elevada concentración de anticuerpos anti-T. cruzi). El punto siguiente en la figura $(\mathrm{DO}=0,367)$ corresponde a una paciente adulta tratada con $\mathrm{Nx}$ a los 23 años de edad, quien permanece con elevados títulos serológicos $(\geq 1 / 128)$ aún 20 años después de finalizado el tratamiento y a pesar de tener $3 \mathrm{Xd}$ posttratamiento donde no se observó presencia de $T$. cruzi. Los 3 valores siguientes - DO 0,306; 0,274 y 0,225 - corresponden a 3 niños tratados con Bz a la edad de 11, 14 y 12 años respectivamente y tienen sólo 2 años de control post-tratamiento. El último valor reactivo para ELISA F29 (DO: 0,220) corresponde al infectado

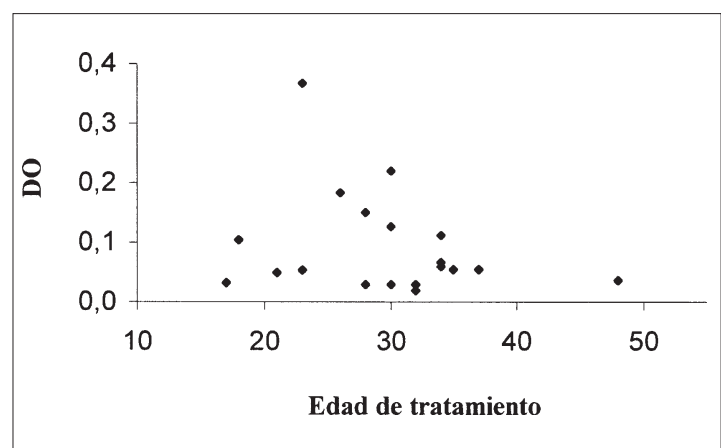

Figura 2. Resultados de ELISA F29 en 19 pacientes adultos con infección crónica del Grupo B, según la edad en que fueron tratados. Grupo B: pacientes tratados con serología convencional reactiva y seguimiento promedio post tratamiento tripanocida de 19,5 $\pm 2,8$ años; DO: lectura en densidades ópticas (valor de corte 0,200); Edad de tratamiento: edad (en años) que recibieron tratamiento tripanocida. adulto tratado con $\mathrm{Bz}$, que desarrolló $\mathrm{MChCr}$ (hemibloqueo anterior izquierdo, bradicardia sinusal y extrasístoles ventriculares frecuentes), sin modificaciones en la silueta cardíaca durante 20 años de seguimiento post-tratamiento. No se encontró el parásito en los $3 \mathrm{Xd}$ realizados durante 10 años posteriores a la finalización de la terapia tripanocida.

Se realizó Xd a 13 pacientes de este grupo; 12/13 de ellos fueron positivos pre-tratamiento. En estos 12 pacientes, los Xd realizados durante 10 años posteriores a la finalización del tratamiento, con un promedio de $3 \mathrm{Xd} /$ paciente, fueron persistentemente negativos.

Grupo C: el test ELISA F29 fue NoR en el $13 \%(7 / 54)$ de los pacientes. En ellos, los títulos de la SC fueron bajos y/o discordantes, permaneciendo sin signos ni síntomas de $\mathrm{MChCr}$ durante 17 años de seguimiento.

Se realizaron $22 \mathrm{Xd}$ a 17 pacientes de este grupo, resultando 4 positivos $(23.5 \%)$, mientras que el test de ELISA F29 fue reactivo en 16 de esos 17 (94,1\%). Los 4 pacientes con Xd positivo presentaron elevada reactividad al ELISA F29.

Todos los infectados con miocardiopatía, al inicio del seguimiento (6 pacientes del grupo $\mathrm{C}$ ) y los que enfermaron durante el mismo (1 paciente del grupo B y 4 pacientes del grupo C), fueron reactivos para ELISA F29.

En las Figuras 2 y 3 están representados los resultados del test de ELISA F29 en los pacientes del grupo B (infectados tratados, con SC reactiva),

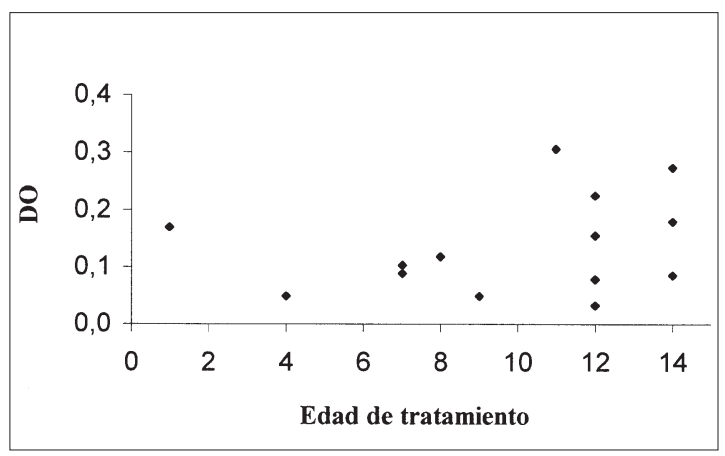

Figura 3. Resultados de ELISA F29 en 14 niños con infección crónica del grupo B, según la edad en que fueron tratados. Grupo B: pacientes tratados con serología convencional reactiva con seguimiento de $2,5 \pm 1,8$ años post-tratamiento tripanocida; DO: lectura en densidades ópticas (valor de corte 0,200); Edad de tratamiento: edad (en años) que recibieron tratamiento tripanocida. 
adultos y niños respectivamente, teniendo en cuenta la edad en que fueron tratados y los años post-tratamiento en que se realizó el control.

En la población adulta, con seguimiento promedio post-tratamiento de $19,5 \pm 2,8$ años, se observa que mientras la SC permaneció reactiva, el ELISA F29 fue NoR en el 89,5\% (17/ 19) (Figura 2).

En el grupo de niños infectados crónicos tratados, con seguimiento post-tratamiento de $2,5 \pm 1,8$ años (excluída la niña aguda congénita), $78,6 \%(11 / 14)$ fueron NoR para ELISA F29. Los 3 niños que permanecieron con ELISA F29 reactiva fueron controlados sólo 2 años después de recibir tratamiento con Bz y además fueron tratados después de los 10 años de edad (Figura 3).

Los resultados obtenidos con el test de ELISA F29 y cada una de las reacciones serológicas convencionales, se analizaron mediante el coeficiente de correlación por rangos de Spearman, ya que las variables no cumplían con los supuestos de distribución normal. En los grupos A y C, analizados en forma conjunta, se halló correlación estadísticamente significativa $(\mathrm{p}<0,05)$, resultando los coeficientes de correlación ( $\mathrm{r}$ ) entre ELISA F29 y AD, IFI, HAI iguales a $0,7788,0,7119$ y 0,7279 respectivamente. En cambio en el grupo B no se observó correlación, ya que mientras la $\mathrm{SC}$ fue reactiva en el $100 \%$, el test de ELISA F29 sólo fue reactivo en $17,6 \%$.

Las Tablas 2 y 3 muestran, respectivamente, los resultados de ELISA F29 y de la SC según la droga tripanocida utilizada en el tratamiento de pacientes con infección crónica. Se aplicó la prueba de $\chi^{2}$ de Pearson, para comparar los resultados de las reacciones serológicas mencionadas, según los pacientes hubieran recibido tratamiento con $\mathrm{Nx}$ o $\mathrm{Bz}$. No se observaron diferencias estadísticamente significativas entre ambas drogas (ELISA F29 $\mathrm{p}=$ 0,678 ; HAI $\mathrm{p}=0,502 ;$ AD $\mathrm{p}=0,677$; IFI $\mathrm{p}=$ $0,697)$.

Además, en estas tablas se observa que en los infectados que recibieron tratamiento tripanocida el test de ELISA F29 resultó negativo en el $91,8 \%$ mientras la SC sólo en el 46\%. Esta

Tabla 2 Resultados del test de ELISA F29 en 61 pacientes con infección crónica, según droga tripanocida utilizada en el tratamiento

\begin{tabular}{lrrrrrr}
\hline \multirow{2}{*}{ ELISA F29 } & & & & Tratados & & \\
& Nx & \% & Bz & \% & Total & \% \\
\hline R & 1 & 5,5 & 4 & 9,3 & 5 & 8,2 \\
NoR & 17 & 94,5 & 39 & 90,7 & 56 & 91,8 \\
Total & 18 & 100 & 43 & 100 & 61 & 100 \\
\hline
\end{tabular}

Se excluyeron 3 niñas tratadas antes del año de edad con infección connatal. R: reactiva; NoR: no reactiva; Nx: nifurtimox; Bz: benznidazol. Al aplicar la prueba $\chi^{2}$ de Pearson, no se observaron diferencias estadísticamente significativas entre los resultados de ELISA F29 según los pacientes hubieran sido tratados con $\mathrm{Nx}$ o Bz ( $\mathrm{p}=$ $0,678)$.

Tabla 3. Resultados de la serología convencional en 61 pacientes tratados con infección crónica, según droga tripanocida utilizada en el tratamiento

\begin{tabular}{lrrrcrr}
\hline SC & Nx & \% & Bz & $\begin{array}{c}\text { Tratados } \\
\mathbf{\%}\end{array}$ & Total & \% \\
\hline R & 11 & 61 & 22 & 51 & 33 & 54 \\
NoR & 7 & 39 & 21 & 49 & 28 & 46 \\
Total & 18 & 100 & 43 & 100 & 61 & 100 \\
\hline
\end{tabular}

Se excluyeron 3 niñas tratadas antes del año de edad con infección connatal. SC: serología convencional; Nx: nifurtimox; Bz: benznidazol. No se observaron diferencias estadísticamente significativas entre los resultados de la serología convencional según los pacientes hubieran sido tratados con $\mathrm{Nx}$ o $\mathrm{Bz}$ aplicando la prueba $\chi^{2} \mathrm{de}$ Pearson $(\mathrm{p}>0,5)$. 
diferencia resultó estadísticamente significativa $\left(\chi^{2}\right.$ de Pearson $\left.=28,5, \mathrm{p}<0,05\right)$.

\section{DISCUSIÓN}

A partir de los resultados de este trabajo, pueden remarcarse aspectos parasitológicos, serológicos y clínicos que indicarían efectos beneficiosos del tratamiento tripanocida en infectados chagásicos crónicos. Desde el punto de vista parasitológico, mediante $\mathrm{Xd}$, aún considerando la baja sensibilidad de este método en el período crónico de la infección, se observó la presencia de $T$. cruzi antes del tratamiento, en cambio no se visualizó el parásito con posterioridad al mismo indicando, al menos, disminución en la carga parasitaria. En cuanto a la respuesta serológica, sólo $8,2 \%$ de los pacientes crónicos tratados fueron reactivos para ELISA F29, mientras esto ocurrió en el $87 \%$ de los infectados que no recibieron tratamiento. Además, en este último grupo, 4/37(10,8\%) pacientes desarrollaron durante el seguimiento manifestaciones clínicas compatibles con $\mathrm{MChCr}$ y ésta patología orgánica fue observada sólo en $1 / 24(4,2 \%)$ del grupo tratado.

Los trabajos realizados por diferentes investigadores ${ }^{18-26}$ para evaluar eficacia del tratamiento tripanocida en pacientes con infección crónica mostraron gran variabilidad en los resultados.

En nuestro Centro de Investigaciones se realizó un estudio de cohorte en niños infectados chagásicos crónicos menores de 14 años, tratados con drogas tripanocidas y no tratados, con un tiempo de seguimiento de hasta 24 años, observándose un 59\% de negativización de la serología convencional en los niños tratados, mientras el grupo de los no tratados mantuvo su serología persistentemente positiva. Las medianas de los tiempos de seroconversión negativa fueron de 3,5 y 8 años para niños que recibieron tratamiento a la edad de 1 a 6 y de 7 a 14 años respectivamente ${ }^{25}$.

También nuestro grupo de trabajo realizó un seguimiento en infectados chagásicos adultos crónicos ${ }^{22,26}$, y observó mejor evolución clínica, disminución de anticuerpos anti-T. cruzi y, seroconversión negativa en el $37 \%$ de los pacientes tratados con un tiempo promedio post-tratamiento de 21 años. Los pacientes que no recibieron tratamiento permanecieron con $\mathrm{SC}$ reactiva.

El criterio de cura después del tratamiento antiparasitario específico es la desaparición de los anticuerpos anti-T. cruzi, medidos por los tests serológicos convencionales ${ }^{27}$. No obstante, la SC presenta limitaciones relacionadas a la elevada complejidad antigénica del T. cruzi, a su variabilidad en las formas evolutivas, a la existencia de diferentes cepas, así como a la similitud con otros parásitos, produciendo desventajas en términos de especificidad, sobretodo en títulos próximos a los de corte. Esto ha generado dificultad para disponer de un antígeno universal que sea reconocido por todos los infectados chagásicos y sólo por ellos ${ }^{28}$.

En el presente trabajo, el $13 \%$ de pacientes que no recibieron tratamiento fueron NoR para el test de ELISA F29. Ellos permanecieron sin signos ni síntomas de MChCr y los resultados de la SC no fueron coincidentes para las distintas reacciones y en los casos en que los tests convencionales fueron reactivos, dicha positividad alcanzó sólo el título de corte. Es preciso realizar estudios complementarios para definir esta discordancia.

En los pacientes crónicos tratados, ya fuera con $\mathrm{Nx}$ o Bz, se observaron diferencias estadísticamente significativas entre el porcentaje de negativización de la SC y ELISA F29: mientras la SC fue NoR en el 46\%, el test de ELISA F29 lo fue en el $91,8 \%$.

A pesar de estas diferencias, se encontró correlación estadísticamente significativa entre los resultados de la SC y ELISA F29 en el grupo de pacientes que no recibieron tratamiento $(C)$ y en el grupo de los tratados con SC NoR (A). En cambio, en los pacientes tratados que mantuvieron positiva la SC (grupo B) no se observó correlación, resultando ELISA F29 reactiva sólo en el $17,6 \%$ de ellos.

Este comportamiento de ELISA F29 en relación a la SC sugiere que este nuevo test podría ser útil para monitorear cambios después del tratamiento tripanocida. Con el diseño utilizado en este trabajo no se puede determinar el tiempo medio de negativización de ELISA F29. A través de un estudio longitudinal, como ya realizamos con la SC, será posible determinar este tiempo y confirmar la presunción que resulta de esta experiencia.

La elevada reactividad al test de ELISA F29 que se observó en los sueros de pacientes en 
quienes se demostró la presencia del parásito, ya sea por Xd o Strout, a pesar del escaso número de muestras, indica una fuerte asociación con la carga parasitaria. Esto permite evaluar tanto falla terapéutica como nivel de infectividad en estudios epidemiológicos.

La proteína F29 se aisló por rastreo inmunológico utilizando suero de conejo antifracción flagelar ${ }^{29}$ en una genoteca de expresión construida a partir de ADN genómico de epimastigotes de T. cruzi (clon miranda 76) en el vector de expresión lambda gt11. Diferentes grupos $^{30-32}$ han aislado previamente esta proteína que está localizada en el flagelo del parásito y es miembro de la superfamilia proteínas de unión al calcio "EF-hand".

La proteína recombinante $\mathrm{F} 29$ fue originalmente clonada en el vector de expresión pMAL p2X (New England BioLabs) ${ }^{12}$. Dicho clonado presentaba dos inconvenientes: escasa expresión y la asociación a la proteína de unión a la maltosa (MBP) que provocaba reacciones serológicas inespecíficas. Con el objetivo de superar ambos problemas, el gen de la proteína F29 fue subclonado en el vector de expresión pQE32. (Qiagen).

El uso de la 6His-F29 en lugar de la MBP-F29 simplificó el ensayo y redujo el costo debido a que el método anterior requería la adición de MBP en la dilución de los sueros para evitar las reacciones inespecíficas.

La producción de proteína F29 es sencilla y escalable, como se demostró en un trabajo conjunto entre el Instituto Nacional de Parasitología y la Universidad de Quilmes (resultados no publicados), lo que permite la aplicación de la ELISA F29 a grandes grupos de población.

\section{RESUMEN}

La proteína flagelar F29 es una proteína ligadora de calcio del Trypanosoma cruzi. En el presente trabajo se realizó un estudio transversal en sueros de pacientes con infección crónica por T. cruzi tratados con nifurtimox $(\mathrm{Nx})$ o benznidazol $(\mathrm{Bz})$ y no tratados, para evaluar el antígeno F29 como marcador de eficacia terapéutica. Se utilizó un ensayo inmunoenzimático con la proteína recombinante F29 (ELISA F29) y se analizó su relación con la serología convencional (SC) y la evolución parasitológica y clínica en esos pacientes. Se estudiaron 118 sueros de pacientes que formaban parte de una cohorte, de los cuales se disponía de información retrospectiva, serológica, parasitológica y clínica.Los pacientes se dividieron en 3 grupos: A) 30 tratados negativizaron SC post-tratamiento; B) 34 tratados permanecieron con SC reactiva; C) 54 no tratados. Las muestras de suero se procesaron a doble ciego en forma simultánea mediante serología convencional (hemoaglutinación indirecta, aglutinación directa e inmunofluorescencia indirecta) y ELISA F29. E1 test ELISA F29 resultó no reactivo en: 100\% del grupo A, $82,4 \%$ del grupo B y $13 \%$ del grupo C. Los infectados con alteraciones electrocardiográficas compatibles con miocardiopatía chagásica crónica $(\mathrm{n}=11)$ fueron reactivos al ELISA F29. Los pacientes en quienes los estudios parasitológicos (xenodiagnóstico y/o strout) fueron $(+)$ presentaron elevada reactividad al ELISA F29. La correlación entre ELISA F29 y $\mathrm{SC}$ en los pacientes tratados con $\mathrm{SC}$ no reactiva (grupo A) y no tratados (grupo C), fue significativa $(p<0,05)$. En cambio, en pacientes tratados que mantuvieron la $\mathrm{SC}$ reactiva (grupo B) el test de ELISA F29 fue reactivo sólo en $17,6 \%$. Estos resultados sugieren que el test ELISA F29, rápido y sencillo, podría ser útil para monitorear cambios post-tratamiento tripanocida.

\section{REFERENCIAS}

1.- MOYA P R, PAOLASSO R D, BLANCO S, et al. Tratamiento de la enfermedad de Chagas con Nifurtimox durante los primeros meses de vida. Medicina (Buenos Aires) 1985; 45: 553-8.

2.- STREIGER M L, FABBRO D L, DEL BARCO M L, et al.,Chagas congénito en la ciudad de Santa Fe. Diagnóstico y tratamiento. Medicina (Buenos Aires) 1995; 55: 125-33.

3.- STOPPANI A. Quimioterapia de la enfermedad de Chagas. Medicina (Buenos Aires) 1999; 59: 147-65.

4.- TOLEDO M, GUILHERME A, SILVA J C. Trypanosoma cruzi chemotherapy with benznidazole in mice inoculated with strains from Parana State and different endemic areas of Brasil. Rev Inst Med Trop São Paulo 1997; 39: 283-90.

5.- CAMPOS R F, GUERREIRO M L, SOBRAL K, et al. Response to chemotherapy with benznidazole of clones isolates from the $21 \mathrm{SF}$ strain of Trypanosoma cruzi (biodeme Type II, Trypanosoma cruzi II). Rev Soc Bras Med Trop 2005; 38: 142-6.

6.- SOSA ESTANI S, SEGURA E L, RUIZ A M, et al. Efficacy of chemotherapy with benznidazole in children in the indeterminate phase of Chagas'disease. Am J Trop Med Hyg 1998; 59: 526-9. 
7.- DE ANDRADE A L S, ZICKER F, DE OLIVEIRA R $\mathrm{M}$, et al. Randomised trial of efficacy of benznidazole in treatment of early Trypanosoma cruzi infection. Lancet 1996; 248: 1407-13.

8.- ANDRADE A L, MARTELLI C M, OLIVEIRA R M, et al. Short report: benznidazole efficacy among Trypanosoma cruzi-infected adolescents after a sixyear follow-up. Am J Trop Med Hyg 2004; 71: 594-7.

9.- RODRIGUES COURA, CASTRO S L DE: A Critical Review on Chagas Disease Chemotherapy. Mem Inst Oswaldo Cruz 2002; 97: 3-24.

10.- LAGUENS R, CABEZA MECKERT P, VIGLIANO C. Patogenia de la miocarditis chagásica crónica humana. Medicina (Buenos Aires) 1999; 59: 63-8.

11.- ENGMAN D, LEÓN J. Pathogenesis of Chagas heart disease: role of autoimmunity. Acta Trop 2002; 81: 123-32.

12.- GUEVARA A G, TAIBI A, ALAVA J, et al. Use of a recombinant Trypanosoma cruzi protein antigen to monitor cure of Chagas disease. Trans of the Royal Soc of Trop Med and Hyg 1995; 89: 447-448.

13. KRAUTZ GM, GALVÃO LM, CANÇADO JR, et al. Use of a 24-kilodalton Trypanosoma cruzi recombinant protein to monitor cure of human Chagas' disease. J Clin Microbiol 1995; 33: 2086-90.

14.- PORCEL B M, BONTEMPI E, HERIKSSON, et al. Trypanosoma rangeli and Trypanosoma cruzi: molecular characterization of genes encoding putative calcium-binding proteins, highly conserved in Trypanosomatids. Exp Parasitol 1996; 84: 387-99.

15.- CERISOLA J A, ROHWEDER R, SEGURA E L, et al. El xenodiagnóstico. Normatización. Instituto Nacional de Diagnóstico e Investigación de la Enfermedad de Chagas (INDIECH), Buenos Aires, Argentina 1974.

16.- BARCLAY C A, CERISOLA J A, LUGONES H, et al. Aspectos farmacológicos y resultados terapéuticos del benznidazol en el tratamiento de la infección chagásica. La Prensa Médica Argentina 1978; 65: 239-44.

17.- Ministerio de Salud y Acción Social y COFESA. Normas para atención médica del infectado chagásico. Buenos Aires, Argentina. Editado por Programa de Salud Humana. Noviembre de 1983.

18.- VIOTTI R, VIGLIANO C, ARMENTI H, SEGURA E L. Treatment of chronic Chagas disease with benznidazole: clinical and serologic evolution of patients with long term follow-up. Am Heart J 1994; 127: 151-62.

19.- PRATA A, MACEDO V, SANTOS I, et al. Tratamento da doença de Chagas pelo nifurtimnox (Bayer 2502). Rev Soc Bras Med Trop 1975; 6: 197-308.

20.- CICHERO J A, SEGURA E, QUATROCHI J C. Evolución clínico-parasitológica y tolerancia a la droga de 33 niños con infección crónica tratados con Bay 2502. Bol Chil Parasitol 1969; 24: 59-62.
21.- FRAGATA-FILHO A, SILVA M, BOAINAIN E. Tratamento etiológico da doença de Chagas nas fases agudas e crônica. Rev Soc Cardiol Estado São Paulo 1994; 4: 192-7.

22.- FABBRO D, ARIAS E, STREIGER M, et al. Evaluación de la quimioterapia específica en infectados chagásicos adultos en fase indeterminada con más de quince años de seguimiento. Rev Fed Arg Cardiol 2001; 30: 496505.

23.- FABBRO DE SUASNÁBAR D, ARIAS E, STREIGER $\mathrm{M}$, et al. Evolutive behavior towards cardiomyopathy of treated (nifurtimox or benznidazole) and untreated chronic chagasic patients. Rev Inst Med Trop São Paulo 2000; 42: 99-109.

24.- CANÇADO J R. Long term evaluation of etiological treatment of chagas disease with benznidazole. Rev Inst Med Trop São Paulo 2002; 44: 29-37.

25.- STREIGER M L, DEL BARCO M L, FABBRO D L, et al. Estudo longitudinal e quimioterapia específica em crianças, com doença de Chagas crônica, residentes em área de laixa endemicidade da República Argentina. Rev Soc Bras Med Trop 2004; 37: 365-75.

26.- FABBRO D L, STREIGER M L, ARIAS E D, et al. Trypanocide treatment among adults with chronic Chagas disease living in Santa Fe city (Argentina), over a mean follow-up of 21 years: parasitological, serological and clinical evolution. Rev Soc Bras Med Trop 2007; 40: 1-10.

27.- CANÇADO J R. Criteria of Chagas Disease Cure. Mem Inst Oswaldo Cruz 1999; 94: 331-5.

28.- LUQUETTI OSTERMAYER A, RASSI A. Diagnosis and treatment of the infection by Trypanosoma cruzi. Mem Inst Oswaldo Cruz 2000; 95: 37.

29.- SEGURA E L, VAZQUEZ C, BRONZINA A, et al. Antigens of the subcellular fractions of Trypanosoma cruzi. II. Flagellar and membrane fraction. J Protozool 1977; 24: 540-3.

30.- GONZÁLEZ A, LERNER T J, HUECAS $\mathrm{M}$, et al. Apparent generation of a segmented mRNA from two separate tandem gene families in Trypanosoma cruzi. Nucleic Acids Res 1985; 13: 5789-804.

31.- ENGMAN D M, KRAUSE K H, BLUMIN J H, KIM K S, KIRCHHOFF L V, DONELSON J E. A novel flagellar $\mathrm{Ca} 2+$-binding protein in trypanosomes. $\mathrm{J}$ Biol Chem 1989; 264: 18627-31.

32.- OUAISSI A, AGUIRRE T, PLUMAS-MARTY B, et al. Cloning and sequencing of a 24-kDa Trypanosoma cruzi specific antigen released in association with membrane vesicles and defined by a monoclonal antibody. Biol Cell 1992; 75: 11-70.

Agradecimientos: El presente trabajo ha sido financiado por presupuesto de la ANLIS Dr. Carlos G. Malbran, el CONICET y CAI+D (Universidad Nacional del Litoral).

\section{Correspondencia a:}

Dirección postal: Diana L. Fabbro. 25 de Mayo 1524,

3000 Santa Fe, Argentina.

Fax Facultad de Bioquímica: 0342-4575206

E-mail: dfabbro@fbcb.unl.edu.ar 